\title{
RETOS E INCERTIDUMBRES EN LA PROTECCIÓN JURÍDICA INTERNACIONAL DE LAS MIGRACIONES AMBIENTALES
}

\author{
Susana Borràs
}

\author{
Recibido: Febrero 1 de 2017 \\ Aprobado: Abril 7 de 2017
}

\section{RESUMEN}

La indefinición jurídica internacional actualmente existente del fenómeno de la "migración ambiental", no hace más que acrecentar la situación de vulnerabilidad y de inseguridad de las personas que migran por modificaciones ambientales. La dificultad de aislar el deterioro ambiental de otras variables que influyen en la migración, como la económica, pero también la protección fragmentada y sectorializada del medio ambiente ajena a las consecuencias del deterioro ambiental sobre los derechos humanos han sido algunos de los principales obstáculos. La urgencia y la necesidad de una respuesta adecuada exigen voluntad política y una cooperación jurídica internacional reforzada para procurar una protección efectiva a los migrantes víctimas del deterioro ambiental y responsabilizar a sus causantes. El presente artículo aporta un análisis minucioso y actualizado de los principales avances producidos en la protección jurídica internacional de la migración ambiental, así como de los retos e incertidumbres, que persisten en su determinación.

Palabras clave: Degradación ambiental, Migración ambiental, Refugiados, Desplazados, Apátridas.

\footnotetext{
* Susana Borràs Pentinat es Profesora de Derecho Internacional Público y Relaciones Internacionales e Investigadora del Centro de Estudios de Derecho Ambiental de Tarragona (CEDAT), Universidad Rovira i Virgili (Tarragona-España). "PROYECTO DE I+D: La constitución climática global: gobernanza y Derecho en un contexto complejo" (CONCLIMA $\square$ DER2016 $\square 80011 \square$ P), (MINECO/FEDER, UE), Programa Estatal de Fomento de la Investigación Científica y Técnica de Excelencia, subprograma Estatal de Generación del Conocimiento, en el marco del Plan Estatal de Investigación Científica y Técnica y de Innovación 2013-2016, efectuada por resolución de 17 de junio de 2015 (BOE de 23 de junio) de la Secretaría de Estado de Investigación, Desarrollo e Innovación (SEIDI), Ministerio de Economía y Competitividad, España.
} 


\title{
CHALLENGES AND UNCERTAINTES IN THE INTERNATIONAL LEGAL PROTECTION OF ENVIRONMENTAL MIGRATION
}

\begin{abstract}
The current international legal uncertainty of the phenomenon of "environmental migration" increases the vulnerability and insecurity of people who migrate due to environmental changes. Some of the main obstacles have been the difficulty of isolating the environmental deterioration from other variables influencing migration, such as the economic one, and the fragmented and sectorial protection of the environment which is excluded from being a consequence of the environmental deterioration on human rights.

The urgency and the need for an adequate response call for political will and stronger international legal cooperation to provide effective protection to migrants who are victims of environmental degradation and to hold those responsible accountable. This article provides a detailed and up-to-date analysis of the main developments within the international legal protection of environmental migration, as well as the challenges and uncertainties that persist in its determination.
\end{abstract}

Key words: Environmental degradation, Environmental migration, Refugees, Displaced, Stateless.

\section{DESAFIOS E INCERTEZAS NA PROTEÇÃO JURÍDICA INTERNACIONAL DAS MIGRAÇÕES AMBIENTAIS}

\section{RESUMO}

A indefinição jurídica internacional atualmente existente do fenómeno da "migração ambiental", não faz mais que acrescentar a situação de vulnerabilidade e de insegurança das pessoas que migram por mudanças ambientais. A dificuldade de isolar a deterioração ambiental de outras variáveis que influem na migração, como a econômica, mas também a proteção fragmentada e setorizada do meio ambiente alheio às consequências da deterioração ambiental sobre os direitos humanos tem sido alguns dos principais obstáculos. A urgência e a necessidade de uma resposta adequada exigem vontade política e uma cooperação jurídica internacional reforçada para procurar uma proteção efetiva às migrantes vítimas da deterioração ambiental e responsabilizar a seus causantes. 
Este artigo mostra uma análise minucioso e atualizado dos principais avanços produzidos na proteção jurídica internacional da migração ambiental, bem como os desafios e as incertezas que persistem na sua determinação.

Palavras-chave: Degradação ambiental, Migração ambiental, Refugiados, Deslocados, Apátridas.

"Todos los seres humanos nacen libres e iguales en dignidad y derechos" Art. 1, Declaración Universal de Derechos Humanos, 1948

\section{INTRODUCCIÓN: REALIDADES HUMANAS OLVIDADAS}

Hace dos décadas no se utilizaba el concepto de migración ambiental. Hoy es un término de uso común, porque la crisis migratoria está estrechamente vinculada a la ambiental. Personas y pueblos enteros se han visto obligados a trasladarse desde su tierra natal, debido a problemas derivados con el ambiente, como desastres naturales y también por otras razones de devastación como son la deforestación, desertificación, inundaciones, o sequías, con la consecuente falta de agua, alimentos y energía, y riesgo de enfermedades, lo que hace que para estas personas, existan pocas o ninguna esperanza de retorno.

Lejos de los desastres naturales, como huracanes, ciclones, erupciones volcánicas y terremotos, que han forzado a muchísimas personas a abandonar sus hogares a lo largo de la historia de la humanidad, es necesario poner especial énfasis a la migración generada por la construcción de los denominados "proyectos de desarrollo", como las represas hidroeléctricas, las industrias extractivas o los cultivos intensivos, así como los grandes accidentes industriales o los conflictos por los recursos naturales, los minerales estratégicos (oro, coltán, etc.), entre otros, que siguen alimentando el número de personas despojadas de su sustento más básico.

Estas personas se encuentran normalmente entre los grupos de población más vulnerables pues, como señala el Informe Mundial sobre Ciencias Sociales (2016) elaborado por la UNESCO, “(...) las personas más pobres y vulnerables son también las que se ven más afectadas por el cambio climático y los trastornos del medio ambiente" (p.10). Es decir, son las poblaciones más vulnerables en los países del Sur Global las que se enfrentan a las peores consecuencias ambientales y las que cuentan con menos recursos para poder hacerle frente, quedando a menudo 
atrapadas en ambientes deteriorados pues carecen incluso de los recursos necesarios para migrar.

Como comentaba al respecto Sami Naïr en una entrevista reciente, "[n]o se habla de ellos porque [...] no hay conciencia real de lo que se nos viene encima" (Ramos, entrevista a Sami Neir, 2016). Sin embargo, en los últimos ocho años aproximadamente, 22,5 millones de personas han sido desplazadas cada año por desastres meteorológicos o relacionados con el clima, lo que equivale a unas 62.000 personas al día (Internal Displacement Monitoring Centre (IDMC), 2015). Según la Organización Internacional para las Migraciones (OIM) sitúa la cifra global en alrededor de 200 millones y que en las próximas cuatro décadas el número de desplazados ambientales llegaría a los mil millones. El Alto Comisionado de las Naciones Unidas para los Refugiados (ACNUR) ha estimado que al menos unas 30 millones de personas fueron desplazadas en el 2013 a consecuencia de alteraciones en el clima y desastres naturales; y que en la actualidad existen alrededor de 50 millones de refugiados en el mundo y en varios casos es posible conectar las causas de su desplazamiento con alteraciones climáticas.

A pesar de que sigue sin existir consenso en relación a las cifras, la magnitud de las estimaciones indica que se trata de un fenómeno de vital importancia, que ha de ser reconocido cuanto antes y que se deben tomar medidas al respecto en todos los ámbitos pertinentes.

Con el objetivo de avanzar hacia el reconocimiento y la protección jurídica de quienes migran por motivos ambientales, en este artículo se realiza, en primer lugar, un análisis de las causas ambientales de las migraciones humanas. En segundo lugar se categorizan las migraciones ambientales según diferentes parámetros, para en tercer lugar profundizar en las limitaciones conceptuales y jurídicas para la protección internacional de quienes migran por motivos ambientales. A continuación, se estudian los avances en el reconocimiento y protección internacional de estas personas, para finalizar con las conclusiones más relevantes.

\section{LAS CAUSAS DE LAS MIGRACIONES AMBIENTALES}

Las migraciones ambientales, así como el resto de movimientos de población, no ocurren por una sola razón. Los motivos para migrar suelen ser complejos e incluir múltiples factores, así como depender de las características del entorno y de las circunstancias particulares de cada persona afectada. La degradación ambiental relacionada con las 132 
migratorias puede estar producida, a su vez, por un amplio abanico de motivos, que pueden tener origen natural o antropogénico y que también se relacionan entre sí.

En relación a las causas naturales de la degradación ambiental, destacan por su importancia los desastres naturales, que engloban actividades geofísicas (terremotos, erupciones volcánicas, avalanchas, deslizamientos de tierra, etc.); actividades meteorológicas (ciclones tropicales, tornados, huracanes, tifones, etc.); y actividades hidrológicas (inundaciones, por ejemplo).

Los desastres naturales generan graves perjuicios humanos, económicos y materiales, y suelen perjudicar en mayor medida a los países menos desarrollados económicamente y, sobre todo, a los que tecnológicamente no disponen de los medios suficientes para detectar los posibles riesgos naturales, ni mucho menos para hacer frente a sus consecuencias devastadoras. Se estima que en 2015, los desastres desplazaron cerca de 19.2 millones de personas, es decir, más del doble del número de desplazamientos generados por conflictos y violencia. (IDMC 2016, p. 7). A modo de ejemplo, en 2016 en Ecuador, el terremoto de 7,8 grados de intensidad en la escala de Richter afectó a unas 750.000 personas y dejó al menos a 22.000 sin hogar. Unos años antes, el terremoto de Haití en 2010 de 7,3 grados, causó la muerte de 222.570 personas, más de 1,5 millones de personas se quedaron sin hogar y sumió en el caos al país más pobre del hemisferio occidental (Bilham, 2010, 878-879). En Nepal, a su vez, se estima que el terremoto de magnitud 7,8 que asoló el país en abril de 2015 causó el desplazamiento de cerca de 2,8 millones de personas (UNOCHA, 2 de junio de 2015). Asimismo, el 26 de diciembre de 2004, un maremoto de 9 grados asoló la costa noroeste de la Isla de Sumatra (Indonesia), las Maldivas y Sri Lanka. Este maremoto, siguiendo a Castillo (2011), resultó en la muerte de casi 300.000 personas y en el desplazamiento de cerca de 2 millones (Castillo, 2011). Estos efectos tan devastadores ponen de manifiesto la vulnerabilidad humana ante los riesgos naturales, así como sus consecuencias desproporcionadas para las poblaciones con menos recursos, especialmente.

Por otro lado, el huracán Katrina, que afectó a los Estados Unidos en agosto del 2005, castigó especialmente la zona de Nueva Orleans, donde reside la población más pobre del país. Como no hubo ningún tipo de planificación previa, este evento causó miles de desplazamientos y murieron miles de personas (OIM, 2009, p.20). Del mismo nivel de intensidad que el huracán Katrina, el huracán Yasi, que cruzó Queensland (Australia), en febrero de 2011 , con vientos de hasta $285 \mathrm{~km} / \mathrm{h}$, afectó gravemente a los edificios más

1 Ver OXFAM. Ecuador Earthquake. Disponible en: <www.oxfam.org/en/emergencies/ecuadorearthquake.28>, acceso el 28 de enero de 2017. 
antiguos. En este caso los sistemas de control y previsión permitieron alertar a la población y efectuar una evacuación de más de 300.000 personas, sin que el paso del huracán registrara ninguna víctima (Europa Press, 2 de febrero de 2011). El maremoto y posterior tsunami que afectó Japón en 2011 causó el desplazamiento de más de 63.000 personas (Oda, 11 de agosto de 2011). Estos son ejemplos de cómo los Estados, aún preparados tecnológicamente para la detección de estos fenómenos y con capacidad de evacuar su población, también pueden verse gravemente afectados por los desastres naturales.

En cuanto a la degradación ambiental de origen antropogénico, es importante resaltar que ésta deriva de las actividades propiamente humanas enraizadas en un modelo de crecimiento económico basado en la explotación insostenible de los recursos naturales y con graves impactos ambientales. Los efectos de la degradación ambiental permanente originan modificaciones ambientales que pueden incluso contribuir en la gravedad de los desastres naturales mencionados anteriormente.

En este grupo de causas antropogénicas de la degradación ambiental pueden distinguirse: la deforestación, las sequías, la degradación del suelo, la contaminación en todas sus formas, la pérdida de biodiversidad, la construcción de proyectos de desarrollo y, entre otras, la explotación desmesurada de los recursos. También se han de destacar las causas de ámbito tecnológico que derivan de accidentes industriales, químicos, nucleares o genéticos y las de ámbito bélico, relacionadas con los conflictos armados o con la violencia generalizada sobre el medio ambiente, es decir, la utilización del medio ambiente como arma de guerra y las consecuencias que provocan este tipo de conflictos sobre el propio medio natural ${ }^{2}$. Asimismo, el control de los recursos naturales se ha convertido más que nunca, a menudo con apoyo extranjero, en un objetivo de las partes en conflicto.

La construcción de grandes obras de infraestructura o, como se suelen denominar, "proyectos de desarrollo" ha generado el desplazamiento de grandes cantidades de población. Durante el siglo XX, entre 40 y 80 millones de personas habían sido desplazadas por las represas en todo el mundo (World Comission on Dams, 2000). Quienes viven río abajo ven sus medios de subsistencia seriamente dañados y se pone en peligro la productividad futura de sus recursos. Además, muchas de las personas

2 Véase HOMER-DIXON, T., On the threshold: environmental change as causes of violent conflict, International Security, v. 16, n. 2, 1991, pp. 76-116.

3 Consultar a BRAUCH, H. G., Environment and human security. Towards freedom from hazard impacts, Bonn: UNU-EHS, Publ., 2005, pp. 25 y ss y, del mismo autor, Threats, challenges, vulnerabilites and risks in environmental and human security, Bonn: EHS, 2005. 
desplazadas no son reconocidas (o registradas como tales) y, por lo tanto, no suelen ser reasentadas o indemnizadas correctamente ${ }^{4}$.

Según la Asociación Interamericana para la Defensa del Ambiente (AIDA, 2009) este tipo de construcciones son comunes en América Latina, donde existen más de 300 proyectos de grandes represas en construcción o planeados. En Brasil, por ejemplo, desde hace 20 años hasta la actualidad, se está librando una complicada lucha ante la inminente construcción de la represa hidroeléctrica "Belo Monte" en el río Xingú, en la Amazonía. De finalizarse la construcción de dicha represa, más de $500 \mathrm{~km}^{2}$ de bosques y terrenos agrícolas serían inundados y al menos 20 mil personas serían desplazadas (AIDA, 2009). En Panamá, desde la década de los '70 hasta la actualidad, la comunidad indígena Ngöbe-Buglé vive amenazada por la construcción de una represa hidroeléctrica en el río Tabasará conocida como "Barro Blanco". Si finalmente se cerraran las compuertas de la represa (construida aproximadamente al $90 \%$ en la actualidad) las tierras de los Ngöbe se inundarían y se acabaría con parte de la cultura de esta comunidad (Felipe, Hofbauer, Mayrhofer y Villavicencio, 2016).

Por su parte, el cambio climático supone una de las más graves causas de degradación ambiental de origen antropogénico que está dando lugar al fenómeno conocido como "migraciones climáticas", un subtipo dentro de las migraciones ambientales. En concordancia con el Grupo Intergubernamental de Expertos sobre el Cambio Climático (IPCC, 2014), a pesar de que el cambio climático afecta a todo el planeta, sus impactos son diferentes según las regiones y la capacidad de resiliencia de los grupos de personas afectadas. Ya hay ejemplos teniendo lugar en algunas regiones de África 5 , como en el Sahel y en el Cuerno de África, en los pequeños Estados insulares del Pacífico (Tuvalu, Kiribati, etc.), en Shishmaref y Kivalina en Alaska ${ }^{6}$, en Asia ${ }^{7}$ y también en América Latina ${ }^{8}$.

4 En este tipo de casos, la vulnerabilidad de las víctimas y los abusos de derechos humanos se agudizan por dos razones: la primera reside en el hecho de que normalmente no se respeta el derecho a la participación de las poblaciones afectadas. La segunda está dada porque el concepto de reparación que se tiene en cuenta para compensar a las personas desplazadas es insuficiente y no suele contemplar el valor de la pérdida cultural que sufren. Al respecto, existe una tendencia generalizada a desvalorizar los saberes locales y suponer que una familia desplazada, por el sólo hecho de recibir una vivienda en otro lugar ha sido debidamente compensada.

5 Véase, por ejemplo, el caso de Gana en: RADEMACHER-SCHULZ, C., SCHRAVEN, B. y MAHAMA, E.Time matters: shifting seasonal migration in Northern Ghana in response to rainfall variability and food insecurity. Climate and Development, v. 6m 2014, p. 646.

6 Véase el caso de Alaska en: BRONEN, R. Alaskan communities' rights and resilience. Forced Migration Review, v. 31,2008, p. 30.

7 Véase ANDERSON, T., SHAMSUDDIHA, M. y DIXIT, A. Climate Change Knows No Borders. An analysis of climate change induced migration, protection gaps and needs for solidarity in South Asia. CANSA, Brot für die Welt y Actionaid, 2016.

8 En Mexico: SÁNCHEZ, I. y otros, Forced migration, climate change, mitigation and adaptive policies in Mexico: Some functional relationships. International Migration, v. 52, 2012, p. 51. En Colombia y en Bolivia véase: CEAM, Migración y cambio climático. El caso de Bolivia y Colombia. CEAM, 2012; DISPLACEMENT SOLUTIONS (DS), Climate displacement and planned relocation in Colombia: the case of Gramalote. DS, 2015. 


\section{LA CATEGORIZACIÓN DE LAS MIGRACIONES AMBIENTALES}

Existen diferentes parámetros o dimensiones que permiten categorizar a las migraciones ambientales. Así, en relación a la dimensión temporal del movimiento, estas pueden ser temporales o permanentes dependiendo de las circunstancias de cada caso concreto.

También se pueden categorizar según Hugo (2011), de acuerdo al grado de voluntariedad, aunque la distinción entre voluntario y forzado es muy complicada porque depende de infinitos factores y casuísticas. La mejor forma de entender el grado de voluntariedad de las migraciones ambientales es visualizándolas a lo largo de una línea continua que va desde un extremo en el que se sitúan los casos en los que la migración tiene un carácter preventivo o más voluntario, al opuesto en el que la única alternativa a no moverse, es la muerte, es decir, el grado máximo de forzamiento.

Otra forma de categorizar las migraciones ambientales, dice Laczo y Piguet (2014), es según su dimensión territorial. Al respecto, la mayoría de estos movimientos tienen lugar a nivel interno o, a lo sumo, a un país vecino. Además, la evidencia empírica demuestra que los éxodos masivos cruzando las fronteras estatales no se esperan al menos en el corto plazo. Asimismo, los movimientos desde las zonas rurales a las urbanas no dejan de aumentar.

En base a todos estos parámetros se pueden delimitar al menos tres categorías de movimientos migratorios por razones ambientales: los migrantes temporales, debido a factores temporales ambientales erupciones volcánicas, terremotos, etc. - y que pueden regresar a su hábitat natural; aquellos migrantes permanentemente por cambios drásticos en su entorno - construcción de presas, contaminación por petroleras, etc. $-\mathrm{y}$ que no pueden regresar y aquellos que han migrado permanentemente en busca de una mejor calidad de vida porque su hábitat es incapaz de proveerles las necesidades mínimas - pérdida de productividad en los suelos, desertificación, etc. ${ }^{10}$

9 Ver: MÁÑEZ, M., SCHWERDTNER, K..; PARAGAY, S. Adaptation under Changing Urban Patterns: The Climatic Perspective of Migration en O.C. RUPPEL, C. ROSCHMANN, Y K. RUPPEL-SCHLICHTING (eds), Climate Change: International Law and Global Governance. Volume II: Policy, Diplomacy and Governance in a Changing Climate. Nomos, 2013.

10 Vid. BRAUCH, H. G. (ed.), Berghof-Stiftung für Konfliktforschung: Facing global environmental change, Berlín; Heidelberg: Springer, 2009; también LONERGAN, S., Global environmental change and human security, GECHS. - Bonn: IHDP, 1999. 


\section{LAS LIMITACIONES ACTUALES DE LA PROTECCIÓN INTERNACIONAL DE LA MIGRACIÓNAMBIENTAL}

Estas realidades humanas que representan el fenómeno de la migración ambiental no son actualmente incluidas en el marco jurídico regulatorio internacional, principalmente debido a limitaciones conceptuales y jurídicas, que han perpetrado la invisibilidad de los migrantes ambientales.

\subsection{Las limitaciones conceptuales}

Uno de los aspectos que mayores debates ha generado en relación a la situación de quienes migran por motivos ambientales está relacionado con el uso de la terminología. Esta cuestión no carece de importancia, puesto que determina el régimen jurídico que debe procurar protección a estas personas.

La terminología empleada inicialmente fue la de "refugiado ambiental", utilizada por Lester Brown en 1976. Posteriormente, en 1985, este concepto fue introducido en un informe del Programa de las Naciones Unidas para el Medio Ambiente elaborado por El- Hinnawi, ${ }^{11}$ profesor del Egyptian National Research Centre, del Cairo y popularizado por la premio Nobel de la Paz Wangari Maathai. Más adelante, el profesor Norman Myers (1995)cuyas cifras de "refugiados ambientales" han sido constantemente reproducidas - empleó a su vez dicha denominación. ${ }^{12}$ El término "refugiado ambiental" fue utilizado por un gran número de autores ${ }^{13} \mathrm{y}$ sigue siendo el más empleado por los medios de comunicación (Vlassopoulos, 2014).

Con el tiempo, se produjo una proliferación y polarización terminológica y conceptual, surgiendo términos como migrante ambiental, y otras denominaciones similares, tales como migración

11 Según este autor: "Environmental refugees are defined as those people who have been forced to leave their traditional habitat, temporary or permanently, because of a marked environmental disruption (natural and/or triggered by people) that jeopardized their existence and/or seriously affected the quality of their life. By 'environmental disruption' in this definition is meant any physical, chemical and/or biological changes in the ecosystem (or the resource base) that render it, temporarily or permanently, unsuitable to support human life". Ver EL-HINNAWI, Environmental Refugees. Nairobi: United Nations Environmental Programme, 1985, p. 4. 12 MYERS, N.; KENT, J. Environmental Exodus: An Emergent Crisis in the Global Arena. Washington DC: Climate Institute, 1995.

13 Vid. EL-HINNAWI, E., op.cit, p. 4; SUHRKE, A., VISENTIN, A., The environmental refugee: a new approach, en Ecodecision, septiembre, 1991, pp. 73-74; SUHRKE, A., "Environmental degradation and population flows", en Journal of International Affairs, 47(2), 1994, pp. 473-496; VLACHOS, E., "International migration and environmental refugees", en RAMPHAL, S., SINDING, S., Population growth and environmental issue, Wesport, Praeger, 1996; KLIOT, N., Environment, Migration and Conflict: A Critical Review, University of Haifa, Israel, 2000; KEANE, D., The Environmental Causes and Consequences of Migration: A Search for the Meaning of Environmental Refugees, en Georgetown International Environmental Law Review, 2004; CASTILLO, J., op. cit. También, BORRAS PENTINAT, S., Refugiados ambientales: el nuevo desafío del derecho internacional del medio ambiente, Revista de Derecho (Valdivia), v. XIX, n. 2, 2006, pp. 85-108, entre otros. 
forzosa ambiental, migración motivada por el medio ambiente, refugiados climáticos, refugiados del cambio climático, personas desplazadas por el clima, refugiados de los desastres naturales, desplazados ambientales, eco-refugiados, climigrantes y personas ecológicamente desplazadas, entre otros tantos ejemplos. Lo cierto es que las diferencias entre estos términos son menos importantes que lo que tienen en común, pues todos sugieren que existe una relación, directa o indirecta, entre la degradación ambiental y la migración.

En la actualidad, gran parte de la doctrina coincide en emplear la denominación "migrante ambiental", independientemente de su concreto estatuto jurídico. Así, la Organización Internacional para las Migraciones (OIM) define migrantes ambientales como:

Las personas o grupos de personas que, por motivo de cambios repentinos o progresivos en el medio ambiente, que afectan adversamente su vida o sus condiciones de vida, se ven obligados a abandonar sus lugares de residencia habituales, o deciden hacerlo, bien sea con carácter temporal o permanente, y que se desplazan dentro de sus propios países o al extranjero. (1 de noviembre de 2007)

Esta definición comprende a las personas que se trasladan por cualquier causa que dé lugar a la degradación ambiental, así como tanto a las que optan por migrar como a las que no les queda otra opción, es decir, es aplicable independientemente de la causa concreta y del grado de voluntariedad de la migración. Asimismo, también reconoce los traslados internos e internacionales.

A pesar de existir cierto consenso entre las numerosas denominaciones para referirse a los movimientos forzados de población en función de su realidad, en parte promovido por la propia OIM, la situación tan extrema como es la de la posible desaparición o inhabitabilidad permanente de los pequeños Estados insulares plantea la posible adecuación de la protección de sus habitantes mediante la aplicación del estatuto de refugiado, como se analiza más adelante y, por tanto, justifica en determinadas ocasiones la utilización de la denominación "refugiado ambiental".

\subsection{Las limitaciones jurídicas de reconocimiento y protección internacional}

Una de las cuestiones más importantes en relación a las migraciones ambientales reside en cómo proteger a estas personas, pues, 
especialmente quienes migran internacionalmente, se encuentran en la actualidad en un limbo jurídico. Así, se plantea la posible aplicación de los regímenes jurídicos de las migraciones forzadas y/o del desplazamiento interno, según si quien migra cruza o no una frontera internacionalmente reconocida, y el de la apatridia, en caso de pérdida de nacionalidad, por ejemplo en los casos de los pequeños Estados insulares amenazados por la elevación del nivel del mar.

\subsubsection{El estatuto de refugiado y la condición de desplazado: Una} posibilidad para proteger a los migrantes ambientales podría resultar en la aplicación de la definición legal de "refugiado" y los derechos de los refugiados regulados en la Convención sobre el Estatuto de los Refugiados de 1951 y su Protocolo de 1967 ("Convención de Ginebra", en adelante). Sin embargo, se dan un sinnúmero de limitaciones que se analizan a continuación.

Así, en relación con el concepto jurídico de refugiado ${ }^{14}$, es necesario señalar, en primer lugar, que la definición sólo se aplica a las personas que ya han cruzado una frontera internacional y, como se analizó anteriormente, la mayoría de las migraciones ambientales tienen lugar a nivel interno. En segundo lugar, existen otras dificultades a la hora de aplicar la definición jurídica de refugiado a los migrantes ambientales. Por ejemplo, es difícil constatar que la degradación ambiental pueda constituir un motivo de persecución, en el sentido jurídico de la palabra, pues la "persecución" implica violaciones de los derechos humanos que son suficientemente graves, debido a su naturaleza inherente o debido a su repetición. ${ }^{15}$ En tercer lugar, según la Convención de Ginebra, los refugiados huyen de su propio gobierno (o de agentes privados de los cuales el gobierno no puede o no quiere protegerlos de ellos), pero una persona que huye de los efectos de la

14 De acuerdo con este acervo jurídico, un "refugiado" se define como alguien que "(...) debido a fundados temores de ser perseguida por motivos de raza, religión, nacionalidad, pertenencia a determinado grupo social u opiniones políticas, se encuentre fuera del país de su nacionalidad y no pueda o, a causa de dichos temores, no quiera acogerse a la protección de tal país; o que, careciendo de nacionalidad y hallándose, a consecuencia de tales acontecimientos, fuera del país donde antes tuviera su residencia habitual, no pueda o, a causa de dichos temores, no quiera regresar a él” Convención sobre el Estatuto de los Refugiados (adoptada el 28 de julio de 1951, entrada en vigor el 22 de abril de 1954) 189 UNTS 137, artículo 1 A(2), y su Protocolo sobre el Estatuto de los Refugiados de 1967 (adoptado el 31 de enero de 1967, entrada en vigor el 4 de octubre de 1967) 606 UNTS 267.

15 Véase también Directiva 2004/83/EC del Consejo de 29 de abril de 2004 por la que se establecen normas mínimas relativas a los requisitos para el reconocimiento y el estatuto de nacionales de terceros países o apátridas como refugiados o personas que necesitan otro tipo de protección internacional y al contenido de la protección concedida [2004], Diario Oficial de la Unión Europea L304/12, artículo 9. Al respecto, el ACNUR organizó una mesa redonda de expertos sobre cambio climático y desplazamiento que se celebró en Bellagio en febrero de 2011. En esta reunión se rechazó el uso de los términos "refugiado climático" y "refugiado ambiental", ya que los consideraron son inexactos y engañosos. Informe del Relator Especial sobre los derechos humanos de los migrantes A/67/299 Sexagésimo séptimo período de sesiones Tema 70 b) del programa provisional "Promoción y protección de los derechos humanos: cuestiones de derechos humanos, incluidos otros medios de mejorar el goce efectivo de los derechos humanos y las libertades fundamentales", 13 de agosto de 2012. Disponible en: www.acnur.es/pdf-reunionexpertos/2011-02-22.25-Resumen.pdf. 
degradación ambiental no escapa necesariamente de su gobierno. En cuarto lugar, en relación con el elemento de la "persecución", la Convención de Ginebra requiere que tal persecución sea por motivos de raza, religión, nacionalidad, opinión política o pertenencia a determinado grupo social. Esta cuestión es también problemática por cuanto los efectos de la degradación ambiental son en gran medida indiscriminados, en lugar de estar vinculados a las características particulares, como los antecedentes de una persona o sus creencias. En consecuencia, es difícil de establecer el argumento de que las personas afectadas por la degradación ambiental pudieran constituir "un determinado grupo social", porque el derecho exige que el grupo debe estar conectado por una característica fundamental, inmutable, que no sea el riesgo de persecución por sí mismo.

No obstante las limitaciones mencionadas, es necesario precisar que la Convención de Ginebra puede aplicarse en situaciones específicas, por ejemplo, cuando "las víctimas de desastres naturales huyen debido a que su gobierno ha retenido u obstruido deliberadamente la asistencia con el fin de castigarlas o marginarlas debido a alguno de los cinco motivos [de la Convención]" (ACNUR, 20 de mayo de 2009, pp. 9-10). Así, siguen existiendo excepciones limitadas donde la exposición al impacto en el clima o la degradación del medio ambiente podría equivaler a persecución por una razón prevista por la Convención. Un ejemplo sería cuando las políticas del gobierno estuvieran dirigidas a determinados grupos que dependen de la agricultura para su supervivencia, donde el cambio climático ya estuviera obstaculizando esa subsistencia. Otro ejemplo sería una hambruna inducida por un gobierno que destruye cultivos, contamine el agua o contribuya a la destrucción del medio ambiente al contaminar la tierra y el agua.

Además de la Convención de Ginebra, a nivel regional el estatuto de refugiado se reconoce en dos instrumentos internacionales más: la Convención de la Organización de la Unión Africana sobre los Refugiados de $1969^{17}$ y la Declaración de Cartagena sobre los Refugiados de $1984^{18}$. Ambas constituyen prominentes instrumentos

16 SERAC; CESR contra Nigeria, Decisión con respecto a la comunicación No ${ }^{\circ} 155 / 96$, ACHPR/COMM/A044/1, XV informe anual de la actividad de la Comisión Africana de Derechos Humanos y de los Pueblos (7 de mayo de 2002), anexo V, párrafo 44. Cooper argumenta que la desertificación en el Sahel africano y la explosión nuclear en Chernóbil constituyeron persecución: véase JB COOPER, Refugiados ambientales: satisfacer los requisitos de la definición de refugiado. Environmental Law Journal de la Universidad de Nueva York, v. 6, 1998, p. 480.

17 Convención de la OUA por la que se regulan los Aspectos Específicos fe Problemas de los Refugiados en África (adoptada 10 septiembre 1969, entró en vigor el 20 de junio de1974) 1001 UNTS 45.

18 Declaración de Cartagena sobre Refugiados (Adoptado por el "Coloquio Sobre la Protección Internacional de los Refugiados en América Central, México y Panamá: Problemas Jurídicos y Humanitarios", celebrado en Cartagena, Colombia, del 19 al 22 de noviembre de 1984) en 'Annual Report of the Inter-American Commission on Human Rights' (1984- 85) OAS Doc OEA/Ser.L/V/II.66/doc.10, rev 1, 190-3. 
regionales sobre refugiados que amplían la definición de refugiado para África y América Latina, proponiendo nuevos enfoques para las necesidades humanitarias de las personas refugiadas y desplazadas, con un espíritu de solidaridad y cooperación. Así, ambas incluyen entre las causas para migrar de manera forzada a las situaciones que han alterado gravemente el orden público ${ }^{19}$, que, de cierta forma, podría resultar equiparable a la degradación ambiental como la causante de hambrunas y sequías. Esta referencia a circunstancias que perturben el orden público ha sido interpretada como clave para incluir los refugiados ambientales. No obstante, la Convención de la OUA solo permite que aquellas personas que sufren "fundado temor a ser perseguidas" califiquen como refugiadas, manteniéndose uno de los inconvenientes señalados en el análisis de la Convención de Ginebra de 1951. A esto se le añade que, por el momento, los principales inconvenientes para ampliar la protección a los migrantes ambientales son que, por un lado, tienen carácter regional, no universal, $\mathrm{y}$, por otro lado, no recogen específicamente los motivos ambientales. Además, la Declaración de Cartagena se trata de un instrumento jurídicamente no vinculante.

La no adecuación del concepto de "refugiado" ha sido confirmada por algunos casos en Australia y Nueva Zelanda, en los que algunos habitantes de Tuvalu y Kiribati habían solicitado el reconocimiento de esta condición de refugiado por los impactos del cambio climático. No obstante, en ninguno de ellos se admitió el reconocimiento de tal condición de refugiado. ${ }^{20}$

Por otro lado, cuando el movimiento migratorio se produce dentro de las fronteras de un mismo país, la protección podría regirse por los Principios Rectores de los Desplazamientos Internos de la ONU de 1998. Así, los Principios Rectores contemplan expresamente la protección de las personas desplazadas ${ }^{21}$ a causa de desastres naturales o provocados por el ser humano y contienen importantes protecciones de los derechos humanos en tales casos. El problema de la aplicación de

19 La Convención de la OUA añade a la definición de persona refugiada que establece Ginebra que se otorgará el estatuto de persona refugiada a las personas obligadas a salir de su país de origen "(...) a causa de una agresión exterior, una ocupación o una dominación extranjera, o de acontecimientos que perturben gravemente el orden público". Y la Declaración de Cartagena también aplica la condición de refugiado a las personas cuya “(...) vida, seguridad o libertad han sido amenazadas por la violencia generalizada, la agresión extranjera, los conflictos internos, la violación masiva de derechos humanos u otras circunstancias que hayan perturbado gravemente el orden público".

20 Ver Apelación de Refugiados No 72189/2000, Autoridad de Apelaciones del Estatuto de Refugiados de Nueva Zelanda, 17 de agosto de 2000, párrafo 13. Ver también 0907346 [2009] RRTA 1168 (10 de diciembre de 2009) párrafo 51 (Tribunal de Revisión de Refugiados de Australia).

21 Según los Principios Rectores, el desplazamiento se produce cuando las "personas o grupos de personas [...] se han visto forzadas u obligadas a escapar o huir de su hogar o de su lugar de residencia habitual, en particular como resultado o para evitar los efectos de un conflicto armado, de situaciones de violencia generalizada, de violaciones de los derechos humanos o de catástrofes naturales o provocadas por el ser humano." 
este instrumento radica en que no es obligatorio y no cubre las migraciones forzadas internacionales. Por su parte, la Convención de Kampala sobre los Desplazados internos de la Unión Africana (2009), si bien es el primer tratado internacional vinculante sobre desplazamiento interno que ofrece importantes protecciones de los derechos humanos para las personas desplazadas debido a factores ambientales, tampoco cubre los migrantes que cruzan fronteras y su ámbito de aplicación es el regional.

42.2. El estatuto de apátrida: La inhabitabilidad progresiva o desaparición permanente del territorio de algunos pequeños Estados insulares de escasa elevación debido al aumento del nivel del mar y a otros efectos del cambio climático representa un panorama poco alentador para la población de estas regiones. Estas circunstancias han impulsado la discusión sobre si a largo plazo podría llegar a considerárseles apátridas en sentido jurídico. ${ }^{22}$

La definición de "apátrida", incluida en el artículo 1 de la Convención sobre el Estatuto de los Apátridas (1954), restringe deliberadamente su alcance a "toda persona que no sea considerada como nacional suyo por ningún Estado conforme a su legislación" (art. 1). ${ }^{23} \mathrm{Al}$ respecto, a pesar de la novedad de la situación de pérdida total del territorio de un Estado, existe una presunción general de continuidad de la condición de Estado y de personalidad jurídica internacional en virtud del Derecho internacional. Así, la condición de Estado no se pierde de forma automática con la pérdida de territorio habitable, ni está necesariamente afectada por los movimientos de población. ${ }^{24}$

En el caso de la posible desaparición o inhabitabilidad de los pequeños Estados insulares, la Convención de 1954 no protegería a sus habitantes a menos que el país en cuestión hubiese retirado formalmente la nacionalidad de las personas, lo cual resulta improbable por las obligaciones impuestas por el derecho de los derechos humanos. No

22 Véase McADAM, J., "Disappearing States", Statelessness and the Boundaries of International Law, en Climate Change and Displacement: Multidisciplinary Perspectives, pp. 105-130; Jane McAdam, compiladora, Hart Publishing, Portland, Oregon, 2010. Consultar ACNUR, Cambio Climático y apatridia: una visión general. Documento presentado para la $6^{\mathrm{a}}$ sesión del Grupo de Trabajo Especial sobre la Cooperación a Largo Plazo (GTE - CLP) según la Convención Marco de las Naciones Unidas sobre el Cambio Climático (CMNUCC), 1 al 12 de junio de 2009. Bonn, Alemania.

23 Convención sobre el Estatuto de los Apátridas (adoptada el 28 de septiembre de 1954, entrada en vigor el 6 de junio de 1960) 360 UNTS 117 (Convenio de 1954), en el artículo 1 inciso 1: "A los efectos de la presente Convención, el término "apátrida" designará a toda persona que no sea considerada como nacional suyo por ningún Estado, conforme a su legislación".

24 De acuerdo con el Derecho internacional, un "Estado" existe si reúne cuatro elementos constitutivos: si un territorio definido tiene una población permanente, un gobierno eficaz y la capacidad de entablar relaciones con otros países. Ver el artículo 1 de la Convención de Montevideo sobre los Derechos y los Deberes de 1933. 
obstante, si un país se reconoce como inexistente, entonces su antigua población estaría cubierta por la definición de "apátrida", siempre que no hubiese adquirido una nueva nacionalidad. Esto obligaría a los países signatarios a proporcionar a estas personas en su territorio los derechos contenidos en dicho tratado. El reconocimiento de esta posibilidad parece ser remota, por distintos motivos: uno, por la novedad de esta situación, que no determina si los Estados estarían dispuestos a considerar que un país preexistente ha "desaparecido" y, dos, que la Convención sólo obliga a los pocos Estados que la han ratificado y, por lo tanto, son escasos los que reconocen este estatuto. Sin embargo, desde organizaciones internacionales como la OIM consideran que la inundación permanente del territorio insular de un Estado podría, potencialmente, forzar a que se empleara la Convención de 1954 para proteger los derechos de las personas nacionales de dichos Estados.

En todo caso, siguiendo a Park (2011) la potencial desaparición de los Estados insulares por los efectos del cambio climático aumenta el riesgo de generar el fenómeno de la apatridia de facto a gran escala, que podría convertirse en apatridia de jure si se considerara que el Estado afectado ha cesado su existencia y en caso de que no hubiesen adquirido otra nacionalidad (por ejemplo, trasladándose a otro país y convirtiéndose en sus ciudadanos). Asimismo, es muy importante aplicar los principios establecidos en el otro pilar de la protección ante la apatridia, es decir, la Convención de Convención para Reducir los Casos de Apatridia, de $1961 .^{25}$

\section{NUEVAS PERSPECTIVAS EN EL RECONOCIMIENTO Y PROTECCIÓN INTERNACIONAL DE LOS MIGRANTES AMBIENTALES: LOS DERECHOS DE LAS PERSONAS Y LOS DEBERES DE LOS ESTADOS}

La situación de las personas que se enfrentan a los riesgos generados por las diferentes formas de degradación ambiental hasta el extremo de comprometer sus derechos más básicos $\mathrm{y}$, en definitiva, su subsistencia, exige, sin duda alguna, una respuesta internacional. Así, la inadecuación del estatuto de refugiado y la incapacidad de los Estados de planificar un proceso migratorio adecuado para sus habitantes, ha exigido plantear respuestas para procurar la protección y la asistencia de las poblaciones que huyen: por una parte, mediante la readaptación y adecuación de la regulación jurídica internacional, otorgando una cobertura jurídica más allá del estatuto de refugiado que, en algunos de los casos, ya existe en el 
ordenamiento jurídico internacional $\mathrm{y}$, por otra, nuevas propuestas basadas en la denominada "protección complementaria", que buscan no solo proteger y visibilizar la situación de los migrantes ambientales, sino también reforzar y reafirmar la debida diligencia, la obligación de proteger a la población y la obligación de cooperar que tienen los Estados en relación con los movimientos de población por causas ambientales.

\subsection{Propuestas de readaptación del marco jurídico internacional}

De las dificultades de aplicar instrumentos internacionales ya existentes surge la idea de crear una nueva convención para proteger de manera explícita a los migrantes ambientales. Un ejemplo es la Convención propuesta por la Universidad de Limoges para regular y proteger a las migraciones forzadas por causas ambientales. ${ }^{26}$ Otro ejemplo lo constituye la "Convention for Persons Displaced by Climate Change", impulsada desde el Instituto de Estudios Avanzados de la Universidad del Oeste de Australia. ${ }^{27}$ No obstante, estas propuestas teóricas presentadas no han contado con el apoyo político necesario.

Otra de las opciones más concretas de articulación de la protección de la migración ambiental, en específico por el cambio climático, lo constituye la adopción un nuevo Protocolo a la Convención Marco de las Naciones Unidas para el Cambio Climático (CMNUCC), denominado "Protocol on Recognition, Protection and Resettlement of Climate Refugees (Climate Refugee Protocol)" (Biermann, 2010, p. 60-88). El principal problema de esta propuesta es determinar el vínculo de causalidad entre la degradación ambiental por causas climáticas y la migración forzada. Además, solo protegería los derechos de un determinado grupo de migrantes ambientales, es decir, los climáticos, dejando de lado la situación de quienes migran por otras causas relacionadas con otras formas de degradación ambiental.

\subsection{La protección complementaria: de los derechos de los migrantes ambientales y del derecho a migrar}

A pesar de las limitaciones de la obligación de protección por parte de un país de acogida, y la falta de reconocimiento de un derecho humano al medio ambiente sano, el derecho de los derechos humanos ha ampliado las obligaciones de protección de los países más allá de la categoría de

26 "Draft Convention on the International Status of Environmentally-Displaced Persons. Los diferentes borradores de la propuesta de convención de Limoges se encuentran disponibles en $<$ www.cidce.org $>$, acceso el 28 de enero de 2017.

27 HODGKINSON, D., BURTON, T., ANDERSON, H.; YOUNG, L. «The Hour When the Ship Comes in»: A Convention for Persons Displaced by Climate Change. Monash University Law Review, v. 36, n. 1, 2010. 
"refugiado", en la medida que las modificaciones ambientales, origen del movimiento forzado, pueden impactar sobre una serie de derechos humanos, cabe citar el derecho a la vida ${ }^{28}$, el derecho a la alimentación adecuada, el derecho a no padecer de hambre ${ }^{29}$, el derecho al agua potable $^{30}$, el derecho al disfrute del más alto nivel de la salud ${ }^{31}$, el derecho a una vivienda adecuada (Pacto Internacional de Derechos Civiles y Políticos, 1966). ${ }^{32}$

La protección más importante e inapelable de quienes migran por motivos ambientales es la asunción necesaria de que los derechos humanos básicos son los que corresponden por igual a toda persona, independientemente de su condición jurídica. El fundamento de la protección de estos derechos, universalmente reconocidos, es la propia dignidad humana ${ }^{33}$. El socorro humanitario debería asegurar que se respetaran los derechos más básicos de los migrantes ambientales, de conformidad con los principios de derechos humanos, y que se prestara la debida atención a los principios fundamentales de no discriminación, participación, empoderamiento y responsabilidad.

Por lo tanto, el Derecho internacional de derechos humanos crea las bases para una "protección complementaria", es decir, una protección basada en los derechos humanos, adicional a la prevista por la Convención de Ginebra y a cualquier otra propuesta de protección que

28 Pacto Internacional de Derechos Civiles y Políticos, (adoptado el 16 de diciembre de 1966, entrada en vigor el 23 de marzo de 1976) 999 UNTS 171, artículo 5; Convención sobre los derechos del niño (adoptada el 20 de noviembre de 1989, entrada en vigor el 2 de septiembre de 1990) 1577 UNTS 3, artículo 6; Declaración Universal de Derechos Humanos (adoptada el 10 de diciembre de 1948) Resolución 217A(III) de la Asamblea General de la ONU, artículo 3.

29 Pacto Internacional de Derechos Económicos, Sociales y Culturales (PIDESC) (adoptado el 16 de diciembre de 1966, entrada en vigor el 3 de enero de 1976) 993 UNTS 3, artículo 11; Convención sobre los derechos del niño artículo 24(c); Convención sobre los derechos de las personas con discapacidad (adoptada el 13 de diciembre de 2006, abierta a la firma el 30 de marzo de 2007) resolución de la Asamblea General de la ONU 61/106 artículos 25(f), 28(1); Declaración Universal de Derechos Humanos, artículo 25.

30 PIDESC, artículos 11 y 12; Convención sobre la eliminación de todas las formas de discriminación contra la mujer (adoptada el 18 de diciembre de 1979, entrada en vigor el 3 de septiembre de 1981) Resolución 34/180 de la Asamblea General, 34 UN GAOR Supp (No. 46) en 193, documento de la ONU A/34/46 (CEDAW), artículo 14(2)(h); Convención sobre los derechos de las personas con discapacidad, artículo 28 (2)(a); Convención sobre los derechos del niño, artículo 24(2)(c).

31 PIDESC artículos 7 (b), 10, 12; CEDAW, artículos 12, 14(2)(b); Declaración Universal de Derechos Humanos, artículo 25; Convención Internacional sobre la eliminación de todas las formas de discriminación racial (adoptada el 21 de diciembre de 1965, entrada en vigor el 4 de enero de 1969) 660 UNTS 195, artículo 5(e)(iv); Convención sobre los derechos del niño, artículo 24; Convención sobre los derechos de las personas con discapacidad, artículos 16(4), 22(2), 25; Convención internacional sobre la protección de los derechos de todos los trabajadores migratorios y de sus familiares, Resolución 45/158 de la Asamblea General de la ONU, de 18 de diciembre de 1990 (Convenio sobre los trabajadores migratorios), artículos 43 (1), 45 (1)(c), 70.

32 PIDESC, artículo 11; Convención sobre los derechos de las personas con discapacidad, artículo 5(e)(iii); CEDAW, artículo 14 (2)(h); Convención sobre los derechos del niño, artículo 27(3); Convenio sobre los trabajadores migratorios, artículo 43(1)(d); Convención sobre los derechos de las personas con discapacidad, artículos 9(1)(a), 28(1), 28(2)(d); Declaración Universal de Derechos Humanos, artículo 25 (1).

33 Ver: PARDO, D. Migración internacional y derechos fundamentales. Disponible en $<$ www.scielo.org.co/scielo.php?script=sci_arttext ypid=S0120-00622012000300024 ylang=es>, acceso el 28 de enero de 2017. 
pueda surgir. Constituye el mínimo común denominador de la dignidad de cualquier persona. Así, en virtud del Pacto Internacional de Derechos Civiles y Políticos de 1966, es preciso que los Estados reconozcan, ante todo y como norma, los derechos civiles y políticos de "todos los individuos en su territorio y sujetos a su jurisdicción, sin distinción" (artículo 2). Además, el Pacto Internacional de Derechos Económicos, Sociales y Culturales, también de 1966, garantiza los derechos sociales, económicos y culturales de todas las personas sin discriminación. Al respecto, esos pactos aseguran la aplicabilidad a los migrantes de los derechos fundamentales (Pardo, s.f). ${ }^{3{ }^{3}}$

En realidad, las respuestas jurídicas no deben basarse necesariamente en nuevas construcciones jurídicas, sino en la adaptación de los marcos jurídicos existentes a los nuevos desafíos producidos por la degradación ambiental. El respeto al derecho a la tierra, a un ambiente saludable y al acceso a los recursos esenciales, como derechos humanos universales, es la base de la protección de los movimientos de personas por causas ambientales. Así, la migración ambiental no deja de ser la consecuencia de una situación de injusticia, de abuso y de violación previa de derechos. Bajo esta concepción, el Derecho internacional de los derechos humanos permite ampliar las obligaciones de protección de los países más allá de la categoría de "refugiado", incluyendo a las personas en riesgo de privación arbitraria de la vida, tortura, tratos o penas crueles, inhumanos o degradantes. Esto se conoce en el Derecho internacional como "protección complementaria", porque describe la protección basada en los derechos humanos que es complementaria a la prevista por la Convención sobre los Refugiados de 1951.

Una de las consecuencias más importantes de esta protección complementaria es la prohibición de devolución a una situación de riesgo real, de privación arbitraria de la vida o de tratos inhumanos o degradantes. Si bien cualquier violación de los derechos humanos podría dar lugar a una obligación de no devolución ${ }^{35}$, en la mayoría de los casos será prácticamente imposible para un solicitante establecer que el control sobre la migración fue desproporcionado en relación con cualquier incumplimiento de un derecho humano (Kacaj, 2002, párr. 26) Por lo tanto aunque se puede intentar una recaracterización del derecho humano violado - por ejemplo, la violación del derecho a un nivel de vida adecuado - como una forma de trato inhumano, que es un derecho que da lugar a la protección internacional, cabe preguntarse si se considera que esas violaciones que no son infligidas por el Estado del que se huye dan

34 Véase la Resolución 2200 A (XXI), anexo, artículos 11 y 12.

35 R c. Juez especial, ex parte Ullah [2004] UKHL 26, párrafos 24 - 25 (Lord Bingham), 49-50 (Lord Steyn), 67 (Lord Carswell). 
lugar a la protección o se consideran malos tratos reconocidos hasta la fecha que dan lugar a una obligación de protección por parte de un tercer Estado (R.c juez especial, Comité de Derechos Humanos, párr. 26).

\subsection{Respuestas políticas y de gobernanza global: construyendo una estrategia de protección integral}

Ante las dificultades o la falta de voluntad de aplicar los regímenes jurídicos existentes a quienes migran por motivos ambientales y de adoptar un nuevo instrumento global, se han desarrollado una serie de propuestas para dar una respuesta adecuada a la situación de estas personas. De entre las que destacan dos: la llamada "Iniciativa Nansen" y los "Principios de Península".

Los Principios Nansen ${ }^{37}$ fueron el principal resultado de la Conferencia Nansen sobre Cambio Climático y Desplazamiento en el Siglo XXI, auspiciada por el Gobierno de Noruega, en Oslo en junio de 2011. Estos Principios contienen un amplio conjunto de recomendaciones y remarcan que las normas de Derecho internacional ya existentes deberían utilizarse plenamente y que se deberían solucionar las lagunas normativas y no solo internacionales, sino también nacionales. Posteriormente y con el propósito de incluir la migración ambiental en la agenda internacional, en octubre de 2012, Noruega y Suiza lanzaron la llamada "Iniciativa Nansen", un proceso consultivo por parte de los Estados que busca construir consenso a la hora de llenar las lagunas de protección legal en el contexto de movimientos de población transfronterizos provocados por desastres repentinos, de degradación lenta y de desastres geofísicos. En octubre de 2015, los Estados participantes en la Iniciativa validaron la versión final de la Agenda de Protección en una consulta global. Esta Agenda no sugiere la creación de ningún tipo de instrumento vinculante de Derecho internacional, pero incluye un número de entendimientos comunes en relación a las migraciones climáticas internacionales e identifica y reitera principios clave en la protección e introduce recomendaciones en el camino a seguir. El propósito de esta Agenda es mejorar la comprensión, proporcionar un marco conceptual e identificar prácticas efectivas para el fortalecimiento de la protección de los movimientos transfronterizos causados por desastres naturales. ${ }^{38}$

36 R c. Juez especial ex parte Ullah [2004] UKHL 26; Comité de Derechos Humanos, Observación General 15: La situación de los extranjeros con arreglo al Pacto (11 de abril de 1986), párrafo 5; véase también Comité de Derechos Humanos, Observación General 18: No discriminación (10 de noviembre de 1989).

37 CICERO, NORWEGIAN MINISTRY OF THE ENVIRONMENT, y NRC. The Nansen Conference. Climate Change and Displacement in the 21st Century. Oslo. Disponible en $<$ www.unhcr.org/4ea969729.pdf>, acceso el 28 de enero de 2017.

38 NANSEN INITIATIVE. The Nansen Initiative. Global Consultation. Conference Report. Ginebra, 12- 13 Octubre de 2015. Disponible en <www.nanseninitiative.org> acceso el 27 de enero de 2017. 
Con posterioridad, otra iniciativa adoptada en este ámbito, fueron los llamados "Principios de Península sobre el Desplazamiento Climático dentro de los Estados", de 2013. "Estos "Principios de Península" se basan en la protección complementaria del régimen internacional de los derechos humanos y se centran en los casos de las personas que se desplazan por motivos principalmente climáticos dentro de un Estado y no a nivel transfronterizo. Establecen, además, las obligaciones de los Estados y de la comunidad internacional hacia estas personas: cumplir con sus obligaciones bajo el Derecho internacional para prevenir y evitar las condiciones que provocan el desplazamiento climático (Principio 5) y prestar asistencia para la adaptación, protección y otras medidas, garantizando la protección contra el desplazamiento climático (Principio 6). Los Principios de Península se pueden emplear como una guía sobre la mejor forma de hacer frente al desplazamiento climático para las propias personas afectadas. ${ }^{40}$

Se ha de resaltar el Acuerdo de París ${ }^{41}$ de 2015 como otro pequeño avance en relación al reconocimiento de quienes migran por motivos ambientales, concretamente por motivos climáticos. Este acuerdo supone un avance significativo en relación a las migraciones inducidas por el cambio climático ya que incorpora en el Preámbulo de manera explícita a las personas migrantes. Además de que se integró una referencia al desplazamiento en las Decisiones para hacer efectivo el Acuerdo, concretamente en "pérdidas y daños". A pesar de esta inclusión, se deja claro en el texto que no habrá ningún tipo de responsabilidad jurídica o indemnización. Aunque no se encuentra en el texto del Acuerdo en sí, al menos se recoge en las decisiones, lo que se ha considerado como un aspecto positivo pero insuficiente en relación a la gravedad y magnitud de las migraciones climáticas. De esta manera, se invita a la creación de un "grupo de trabajo", cuyas tareas serán desarrollar recomendaciones "para evitar, reducir al mínimo y afrontar los desplazamientos relacionados con los efectos adversos del cambio climático" (Informe de la conferencia de las Partes sobre su 21 periodo de sesiones, 2015, p. 9). A pesar de que el acuerdo final no es tan

39 Texto completo disponible en: Los Principios de Península sobre el Desplazamiento Climático dentro de los Estados. Disponible en: <http://displacementsolutions.org/wp-content/uploads/Peninsula-PrinciplesSpanish.pdf>, acceso el 27 de enero de 2017.

40 En abril de 2015 la asociación "Displacement Solutions" editó una guía de bolsillo en un lenguaje menos técnico para que pudiera ser empleada por las propias personas involucradas en el desplazamiento, incluyendo los pasos que deben seguir para conseguir ayuda en caso de desplazamiento climático. Véase DISPLACEMENT SOLUTIONS. The Rights of Climate Displaced Persons. A Quick Guide. Disponible en $<$ http://displacementsolutions.org/wp-content/uploads/2015/04/Pocket-Guide-on-Rights-of-CDPs.pdf $>$, acceso 27 de enero de 2017.

41 Informe de la Conferencia de las Partes 21 período de sesiones. París, 30 de noviembre a 11 de diciembre de 2015. Aprobación del Acuerdo de París. Doc. FCCC/CP/2015/L.9. París. 
ambicioso como debiera, al menos ahora existe un pacto entre casi 200 países en el que se comprometen a tratar el asunto de las migraciones vinculadas al cambio climático. ${ }^{42}$

Más recientemente, los Jefes de Estado y de Gobierno y altos representantes, reunidos en la Sede de las Naciones Unidas en Nueva York adoptaron la llamada "Declaración de Nueva York sobre los Refugiados y Migrantes", de 19 de septiembre de 2016. Este documento representa un paso importante en la realización de un pacto mundial para una migración segura, regular y ordenada, independientemente de las causas que han generado el movimiento migratorio. Los Estados manifiestan su compromiso de avanzar hacia este marco integral, mediante la elaboración de directrices sobre el trato de los migrantes en situaciones de vulnerabilidad y de lograr un reparto más equitativo de la carga y la responsabilidad de acoger y dar apoyo a los refugiados del mundo mediante la aprobación de un pacto mundial sobre refugiados en 2018. Este enfoque omnicomprensivo de la realidad migratoria y del refugio, aunque propiciada por la crisis humanitaria de Siria, ha motivado una respuesta internacional -a modo de declaración políticaante la migración desde una perspectiva, por fin, humanitaria y basada en el compromiso de 193 Estados de procurar protección y garantizar los derechos humanos. ${ }^{43}$ Esto sin duda, constituye, como lo fue en su momento la Declaración Universal de Derechos Humanos, el cimiento de un cambio revolucionario.

\section{CONCLUSIONES: SOBREVIVIR A LAS MODIFICACIONES AMBIENTALES}

Las propuestas jurídicas analizadas se dirigen a encontrar respuestas a una realidad internacional compleja, pero creciente y preocupante como son las migraciones por modificaciones ambientales, que en la actualidad ningún marco jurídico da una respuesta satisfactoria. La verdad es que la solución más adecuada no debería venir necesariamente por una novedad jurídica, sino de una voluntad política de cooperar y de establecer un marco de protección adecuado y suficiente para prevenir

42 UK CLIMATE CHANGE AND MIGRATION COALITION. Paris - Displacement and Migration. What happens next? Disponible en $<\mathrm{http}$ ://climatemigration.org.uk/alex_randall-paris-displacement-and-migrationwhat-happens-next/>, acceso el 30 de enero de 2017

43 Los antecedentes más inmediatos de este enfoque basado en derechos humanos se produjo en 2012, cuando la Asamblea General solicitó a los Estados Miembros, al sistema de las Naciones Unidas, a las organizaciones internacionales, a la sociedad civil y a todas las instancias pertinentes, en especial a la Alta Comisionada de las Naciones Unidas para los Derechos Humanos, al Relator Especial sobre los derechos humanos de los migrantes y al Grupo Mundial sobre Migración, que velarán por que en el Diálogo de Alto Nivel sobre la Migración Internacional y el Desarrollo de 2013, se "analice el vínculo entre la migración y el desarrollo de una manera equilibrada e integral que incluya, entre otras, la perspectiva de derechos humanos" (A/RES/67/172). 
y/o responder a las crisis humanitarias generadas por la degradación del medio ambiente.

Como muy bien explica Sami Naïr "el imperativo moral de resignificar el concepto de refugiado ya no tiene excusas de dilación" (2016, p. 30). En otras palabras, existe el convencimiento en algunos casos concretos de migraciones ambientales, en los que la Convención de Ginebra sí que sería el instrumento adecuado para salvaguardar los derechos de las personas afectadas.

Además de las lagunas jurídica a las que se enfrentan los migrantes ambientales, la inexistencia de una institución internacional que asuma la responsabilidad de las personas que migran de empeora el panorama. El ACNUR, a pesar de reconocer la gravedad de la situación, no ha asumido la protección para las personas que migran por motivos climáticos ni ha revisado su mandato para incluirlos.

La realidad muestra un desacoplamiento de los marcos jurídicos, políticos e institucionales condicionada, ciertamente, por la complejidad y amplitud del fenómeno de la migración ambiental, aunque si bien es verdad no contribuyen para nada a dar respuestas eficaces a la movilidad humana forzada por causas ambientales. La indiferencia de la degradación ambiental, como factor que afecta gravemente al bienestar humano, no hace más que confirmar la necesidad de profundizar críticamente en los estudios sobre los efectos en las migraciones internacionales e implementar políticas reales, que estén dirigidas a la raíz de los problemas. Medidas de prevención, adaptación, de reacción temprana, de resiliencia, de control de determinadas actividades altamente impactantes sobre el medio ambiente, entre otras, no dejan de ser la clave para iniciar una protección integral de los derechos sociales y ambientales, de poblaciones afectadas por las consecuencias de la degradación ambiental.

En todo caso, será interesante ver cómo las distintas iniciativas analizadas van evolucionando, sin dejar de contemplar que la realidad de millones de personas en el mundo es la de huir y sobrevivir a las modificaciones ambientales.

\section{REFERENCIAS BIBLIOGRÁFICAS}

ACNUR (2009a), "Cambio Climático y apatridia: una visión general”. Documento presentado para la $6^{\mathrm{a}}$ sesión del Grupo de Trabajo 150

Especial sobre la Cooperación a Largo Plazo (GTE - CLP) según la 
Convención Marco de las Naciones Unidas sobre el Cambio Climático (CMNUCC), 1 al 12 de junio de 2009. Bonn, Alemania.

ACNUR (2009b). "Desplazamiento forzado en el contexto del cambio climático: Desafíos para los Estados en virtud del derecho internacional", presentado en la sexta reunión del Grupo de Trabajo Especial sobre la cooperación a largo plazo en el marco de la Convención, 20 de mayo de 2009, 9-10.

AIDA. (2009). Grandes Represas en América. ¿Peor el remedio que la enfermedad? Principales consecuencias ambientales y en los derechos humanos y posibles alternativas. Recuperado de www.aida-ameri cas.org/ sites/default/files/InformeAIDA_GrandesRepreseas_Baja Res.pdf

AIDA. (s. f.). La represa hidroeléctrica Belo Monte. Recuperado de www.aida-americas.org/es/project/belomonte

Anderson, T., Shamsuddiha, M. \& Dixit, A. (2016). Climate Change Knows No Borders. An analysis of climate change induced migration, protection gaps and needs for solidarity in South Asia, CANSA, Brot für die Welt \& Actionaid.

Biermann, F., \& Boas, I. (2010a). Preparing for a Warmer World: Towards a Global Governance System to Protect Climate Refugees. Global Environmental Politics, 10(1), 60-88.

Bilham, R. (2010). Haiti Earthquake may have primed Nearby Faults for Failure. Nature, 463 (February), 878-879.

Borras, S. (2006). Refugiados ambientales: el nuevo desafío del derecho internacional del medio ambiente, Revista de Derecho (Valdivia), 10 (2), 85-108.

Brauch, H. G. (1999). Berghof-Stiftung für Konfliktforschung: Facing global environmental change, Berlín; Heidelberg: Springer, 2009; también Lonergan, S., Global environmental change and human security, GECHS. - Bonn: IHDP.

Brauch, H. G. (2005). Environment and human security. Towards freedom from hazard impacts, Bonn: UNU-EHS, Publ., 25 y ss. 
Brauch, H. G. (2005). Threats, challenges, vulnerabilites and risks in environmental and human security, Bonn: EHS.

Bronen, R. (2008). Alaskan communities' rights and resilience, 31 Forced Migration Review 30.

Castillo, J. (2011). Migraciones Ambientales: Huyendo de la Crisis Ecológica en el siglo XXI (1 a ed.). Barcelona: Virus editorial.

Centro de Estudios Amazónicos (CEAM), Migración y cambio climático. El caso de Bolivia y Colombia. (CEAM 2012)

CICERO, Norwegian Ministry of the Environment, \& NRC. (2011). The Nansen Conference. Climate Change and Displacement in the 21st Century. Oslo. Recuperado de www.unhcr.org/4ea969729.pdf

CISC y IED. Informe Mundial sobre Ciencias Sociales 2016. UNESCO, 2016, 10.

Cooper, J. B. (1998). Refugiados ambientales: satisfacer los requisitos de la definición de refugiado. 6 Environmental Law Journal de la Universidad de Nueva York 480.

CRIDEAU, CRDP y OMIJ. (2014). Réseau Déplacés Environnementaux. Recuperado de www.cidce.org/

Displacement Solutions (DS) (2015). Climate displacement and planned relocation in Colombia: the case of Gramalote.

Displacement Solutions. (2015). The Rights of Climate Displaced Persons. A Quick Guide. Ginebra. Recuperado de http://displacement solutions.org/wp-content/uploads/2015/04/ Pocket-Guide-onRights-of-CDPs.pdf

El-Hinnawi, E. (1985). Environmental Refugees, United Nations Environment Programm, Nairobi, Kenya, 4 ss.

Felipe, B., Hofbauer, J., Mayrhofer, M., \& Villavicencio, P. (2016). Rethinking the Role of Development Banks in Climate Finance: Panama's Barro Blanco CDM Project and Human Rights. Law, Environment \& Development Journal, 12 (1). 
Hodgkinson, D., Burton, T., Anderson, H., \& Young, L. (2010). «The Hour When the Ship Comes in»: A Convention for Persons Displaced by Climate Change. Monash University Law Review, 36(1).

Homer-Dixon, T. (1991). "On the threshold: environmental change as causes of violent conflict”, International Security, 16(2), 76-116.

Hugo, G. (2011). Future Demographic Change And Its Interactions With Migration And Climate Change. Global Environmental Change, 21(SUPPL. 1), 21-33.

IDMC, NRC. (2015). Global Estimates 2015. People Displaced by Disasters. Recuperado de http://internal-displacement.org/assets/ library/Media/201507-globalEstimates-2015/20150713-globalestimates-2015-en-v1.pdf

IDMC, NRC. (2016). Global Report on Internal Displacement. GRID 2016. 7. Recuperado de www.internal-displacement.org/assets/ publications/2016/2016-global-report-internal-displacementIDMC.pdf

IPCC. (2014). Cambio climático 2014. Impactos, adaptación y vulnerabilidad. Resumen para responsables de políticas. Suiza. Recuperado de http://ipcc.ch/pdf/assessment-report/ar5/syr/ SYR_AR5_FINAL_full_es.pdf

Keane, D. (2004). The Environmental Causes and Consequences of Migration: A Search for the Meaning of Environmental Refugees, en Georgetown International Environmental Law Review.

Kliot, N. (2000), Environment, Migration and Conflict: A Critical Review, University of Haifa, Israel.

Laczo, F., \& Piguet, E. (2014). Regional Perspectives on Migration, the Environment and Climate Change. En E. Piguet \& F. Laczo (Eds.), People on the Move in a Changing Climate. The Regional Impact of Environmental Change on Migration. (1ra ed.). Nueva York: Springer, 1-20.

Los Principios de Península sobre el Desplazamiento Climático dentro de los Estados. (18 de agosto de 2013). Recuperado de http://displacementsolutions.org/wp-content/uploads/PeninsulaPrinciples-Spanish.pdf 
Máñez, M., Schwerdtner, K. \& Paragay, S. (2013). Adaptation under Changing Urban Patterns: The Climatic Perspective of Migration, en O.C. Ruppel, C. Roschmann, y K. Ruppel-Schlichting (eds.), Climate Change: International Law and Global Governance. 2: Policy, Diplomacy and Governance in a Changing Climate, Berlín: Nomos.

Mcadam, J. (2010). "Disappearing States", Statelessness and the Boundaries of International Law, en McAdam, J (comp.), Climate Change and Displacement: Multidisciplinary Perspectives, 105-130. Portland, Oregon: Hart Publishing.

Myers, N., \& Kent, J. (1995). Environmental Exodus: An Emergent Crisis in the Global Arena. Washington DC: Climate Institute.

Naïr, S. (2016). Refugiados. Frente a la catástrofe humanitaria, una solución real. (1 ra ed.). Barcelona: Crítica, 30.

Naciones Unidas, Asamblea General "Declaración de Nueva York para los Refugiados y los Migrantes". A/71/150 (13 de septiembre de $2016)$. Recuperado de http://www.un.org/es/comun/ ocs/?symbol=A/71/L.1

Naciones Unidas, Convención marco sobre el Cambio Climático. Informe de la Conferencia de las Partes 21 período de sesiones. París, 30 de noviembre a 11 de diciembre de 2015. Doc. FCCC/CP/2015/L.9. París.

Nansen Initiative (2015). The Nansen Initiative. Global Consultation. Conference Report. Ginebra, 12- 13 Octubre de 2015. Ginebra. Recuperado de www.nanseninitiative.org/

Oda, T. (2011). Grasping the Fukushima Displacement and Diaspora. The 2011 East japan Earthquake Bulletin. Tokyo. Recuperado de http://tohokugeo.jp/articles/e-contents24.pdf

OIM. (1 de noviembre de 2007). Nota para las deliberaciones: la migración y el medio ambiente. MC/INF/288. Nonagésima cuarta reunión.

OIM. (2009a). Compendium of IOM's Activities in Migration, Climate Change and the Environment. Ginebra. Recuperado de http://publications.iom.int/es/system/files/pdf/compendium_of_iom s_activities.pdf p. 20). 
OIM. (2014a). IOM Outlook on Migration, Environment and Climate Change. Ginebra. Recuperado de http://publications.iom.int/ system/files/pdf/mecc_outlook.pdf

OXFAM. (2016). Ecuador Earthquake. Recuperado de www.oxfam.org/en/emergencies/ecuador-earthquake

Pardo, D. (2012). "Migración internacional y derechos fundamentales". Ideas y valores. Recuperado de www.scielo.org.co/scielo.php? script=sci_arttext\&pid=S0120-00622012000300024\&lang=es.

Park, S. (2011). Cambio climático y el riesgo de apatridia: La situación de los 'Estados insulares en hundimiento, Series de Investigación: Política de Protección y Asesoría Legal. División de Protección Internacional (ACNUR), Ginebra, 33.

Rademacher-Schulz, C., Schraven, B. y Mahama, E. (2014). Time matters: shifting seasonal migration in Northern Ghana in response to rainfall variability and food insecurity, 6 Climate and Development 46.

Ramos, I. (2016). «La mayoría ya son refugiados medioambientales» Entrevista a Sami Naïr. La Vanguardia. Barcelona. Recuperado de www.lavanguardia.com/internacional/20160925/41552073365/lamayoria-ya-son-refugiados-medioambientales.html

Sánchez, I. y otros (2012). 'Forced migration, climate change, mitigation and adaptive policies in Mexico: Some functional relationships' 51 International Migration 53.

Stoett, P. J. (1993). Environmental refugees: conceptual problems and international mitigation.

Suhrke, A. (1994). "Environmental degradation and population flows", en Journal of International Affairs, 47(2), 473-496.

Suhrke, A., \& Visentin, A. (1991). "The environmental refugee: a new approach", en Ecodecision, septiembre, 73-74.

UK Climate Change and Migration Coalition. (2015). Paris Displacement and Migration. What happens next?. Recuperado de http://climatemigration.org.uk/alex_randall-paris-displacementand-migration-what-happens-next/ 
UNOCHA. (2015). Nepal Earthquake. Flash Appeal for Response to the Nepal Earthquake. Nueva York. Recuperado de http://reliefweb.int/ sites/reliefweb.int/files/resources/nepal_flash_appeal.pdf

Vlachos, E. (1996). International migration and environmental refugees, en Ramphal, S., Sinding, S., Population growth and environmental issue, Wesport, Praeger.

Vlassopoulos, C.A. (2013). Defining Environmental Migration in the Climate Change Era: Problem, Consequence or Solution? En Faist T. \& Schade J. (eds.). Disentangling Migration and Climate Change, (1ra ed.), 145-165. Bielefeld: Springer.

WCD. (2000). Dams and Development: A New Framework for Descision-Making. Reino Unido: Earthscan. Recuperado de www.internationalrivers.org/files/attached-files/world commission_on_dams_final_report.pdf 